\title{
Updated Higgs cross section at approximate $\mathrm{N}^{3} \mathrm{LO}$
}

\author{
Marco Bonvini ${ }^{a}$, Richard D. Ball $^{b}$, Stefano Forte ${ }^{c}$, \\ Simone Marzani ${ }^{d}$ and Giovanni Ridolfi ${ }^{e}$ \\ ${ }^{a}$ Deutsches Elektronen-Synchroton, DESY, \\ Notkestraße 85, D-22603 Hamburg, Germany \\ ${ }^{b}$ Tait Institute, University of Edinburgh, \\ JCMB, KB, Mayfield Road, Edinburgh EH9 3JZ, Scotland \\ ${ }^{c}$ Dipartimento di Fisica, Università di Milano and INFN, Sezione di Milano, \\ Via Celoria 16, I-20133 Milano, Italy \\ ${ }^{d}$ Institute for Particle Physics Phenomenology, Durham University, \\ South Road, Durham DH1 3LE, England \\ ${ }^{e}$ Dipartimento di Fisica, Università di Genova and INFN, Sezione di Genova, \\ Via Dodecaneso 33, I-16146 Genova, Italy
}

\begin{abstract}
:
We update our estimate of the cross section for Higgs production in gluon fusion at nextto-next-to-next-to-leading order $\left(\mathrm{N}^{3} \mathrm{LO}\right)$ in $\alpha_{s}$ in view of the recent full computation of the result in the soft limit for infinite top mass, which determines a previously unknown constant. We briefly discuss the phenomenological implications. Results are available through the updated version of the ggHiggs code.
\end{abstract}


The cross section for the production of a Higgs boson in gluon-gluon fusion (the dominant production subprocess at available collider energies) has been computed up to next-to-next-to-leading order (NNLO) in perturbative Quantum Chromodynamics (QCD) 1], and a calculation of the $\mathrm{N}^{3} \mathrm{LO}$ correction is under way 2,3 . In a recent paper [4] we have provided an estimate of the $\mathrm{N}^{3} \mathrm{LO}$ correction, based on the knowledge of the analytic structure of the coefficients of the perturbative expansion in the space of the variable $N$, Mellin conjugate to $z=m_{H}^{2} / \hat{s}$ where $m_{H}$ is the Higgs mass and $\hat{s}$ is the squared partonic center-of-mass energy. Such knowledge, in turn, originates from resummation of powers of $\log N$ in the large- $N$ regime (soft-gluon or threshold resummation), and from high-energy resummation, which fixes the behavior of the coefficient in the vicinity of its rightmost singularity in the $N$ complex plane. High-energy resummation turns out to have a relatively small direct numerical impact, but its analyticity properties affect considerably the form of soft-emission logarithmic terms.

The result of Ref. [4] in particular included all contributions to the $\mathcal{O}\left(\alpha_{s}^{3}\right)$ which do not vanish as $N \rightarrow \infty$, and which either grow logarithmically, or are constant. These contributions were all known, with the exception of the constant, which in $z$ space corresponds to the coefficient of the $\delta(1-z)$ contribution to the cross section. Recently [3], the full cross section was determined in the soft limit, including this constant, and our approximate result can be updated accordingly: the result of Ref. [3] effectively amounts to a determination of the coefficient $g_{0,3}$ of Ref. [4]. The value of this coefficient was estimated in Ref. [4] to be $g_{0,3}=114.7$ (for finite $m_{t}$ ). This estimate was arrived at by rewriting $g_{0, n}=\bar{g}_{0, n}+r_{n}$, with $r_{3}$ known in terms of available information, noting that the perturbative behaviour of the known coefficients suggests $r_{3} \gg \bar{g}_{0,3}$, and thus simply assuming $g_{0,3}=r_{3}$. Similar estimates for $g_{0}$ have also been obtained using methods for the all-order resummation of constant contributions [5].

In Ref. 3 the coefficient $g_{0,3}$ is determined in the pointlike limit. We wish to use this result while retaining the full $m_{t}$ dependence of all the remaining information (which in particular is important in order to have the correct analytic structure in the high-energy limit). Note that the $m_{t}$ dependence of this constant at previous orders is negligible: at NLO the variation of $g_{0,1}$ when going from finite $m_{t}$ to the pointlike limit is by about $0.5 \%$, and at NNLO the variation of $g_{0,2}$ is by about $1 \%$. At $\mathrm{N}^{3} \mathrm{LO}$ we have a certain latitude in deciding which coefficient we should evaluate in the pointlike limit. We choose to take the pointlike limit value of the coefficient of the delta function in the full $\mathcal{O}\left(\alpha_{s}^{3}\right)$ cross section (as given in Eq. (4) of Ref. [3]).

With this procedure we thus determine $g_{0,3}=116.7$, very close to the value $g_{0,3}=114.7$ used in Ref. [4]. Using the resulting value for $\bar{g}_{0,3}=g_{0,3}-r_{3}=116.7-114.7=2.0$ in Eqs. $(4.1,4.2)$ of Ref. [4] we get the approximate updated total cross section at the Large Hadron Collider (LHC), with $\sqrt{s}=8 \mathrm{TeV}$ and $m_{H}=125 \mathrm{GeV}$, computed using the NNLO NNPDF2.1 [6] set of parton distribution functions with $\alpha_{s}\left(m_{Z}^{2}\right)=0.119$ (i.e. the same choices as in Ref. [4])

$$
\begin{aligned}
\sigma_{\text {approx }}^{\mathrm{N}^{3} \mathrm{LO}}\left(s, m_{H}^{2}\right) & =(22.42 \pm 0.31) \mathrm{pb} & & \text { for } \mu_{\mathrm{R}}=m_{H} \\
& =(23.70 \pm 0.54) \mathrm{pb} & & \text { for } \mu_{\mathrm{R}}=m_{H} / 2,
\end{aligned}
$$

where $\mu_{\mathrm{F}}=m_{H}$, and the error shown is our estimate of the uncertainty in our approximation. The result is extremely close to the value of Ref. [4], the difference being at the permille level.

Our result, and its dependence on the renormalization scale $\mu_{\mathrm{R}}$, are shown in Fig. 1 (the dependence on the factorization scale is negligible, as discussed in Ref. [4]), compared to the lower-order results. Our estimate of the $\mathrm{N}^{3} \mathrm{LO}$ cross section is the red dot-dashed curve; the band provides our estimate for the uncertainty involved in the approximation procedure, details are given in Ref. 4 .

We thus confirm, now on a firmer footing, the conclusion of Ref. [4], namely that the $\mathrm{N}^{3} \mathrm{LO}$ contribution leads (for $\mu_{\mathrm{R}}=m_{H}$ ) to an increase by about $16 \%$ of the NNLO cross section. Note that it was shown in Ref. [7] that this conclusion would very likely be unaffected by the consistent use of $\mathrm{N}^{3} \mathrm{LO}$ parton distributions. This result can be compared to the main commonly-used approximation to higher-order corrections, namely that from threshold resummation at next-to-next-to-leading log (NNLL) [8]. The truncation of the latter to $\mathcal{O}\left(\alpha_{s}^{3}\right)$ is also shown in Fig. 1: it is seen to lead to an 
Higgs cross section: gluon fusion

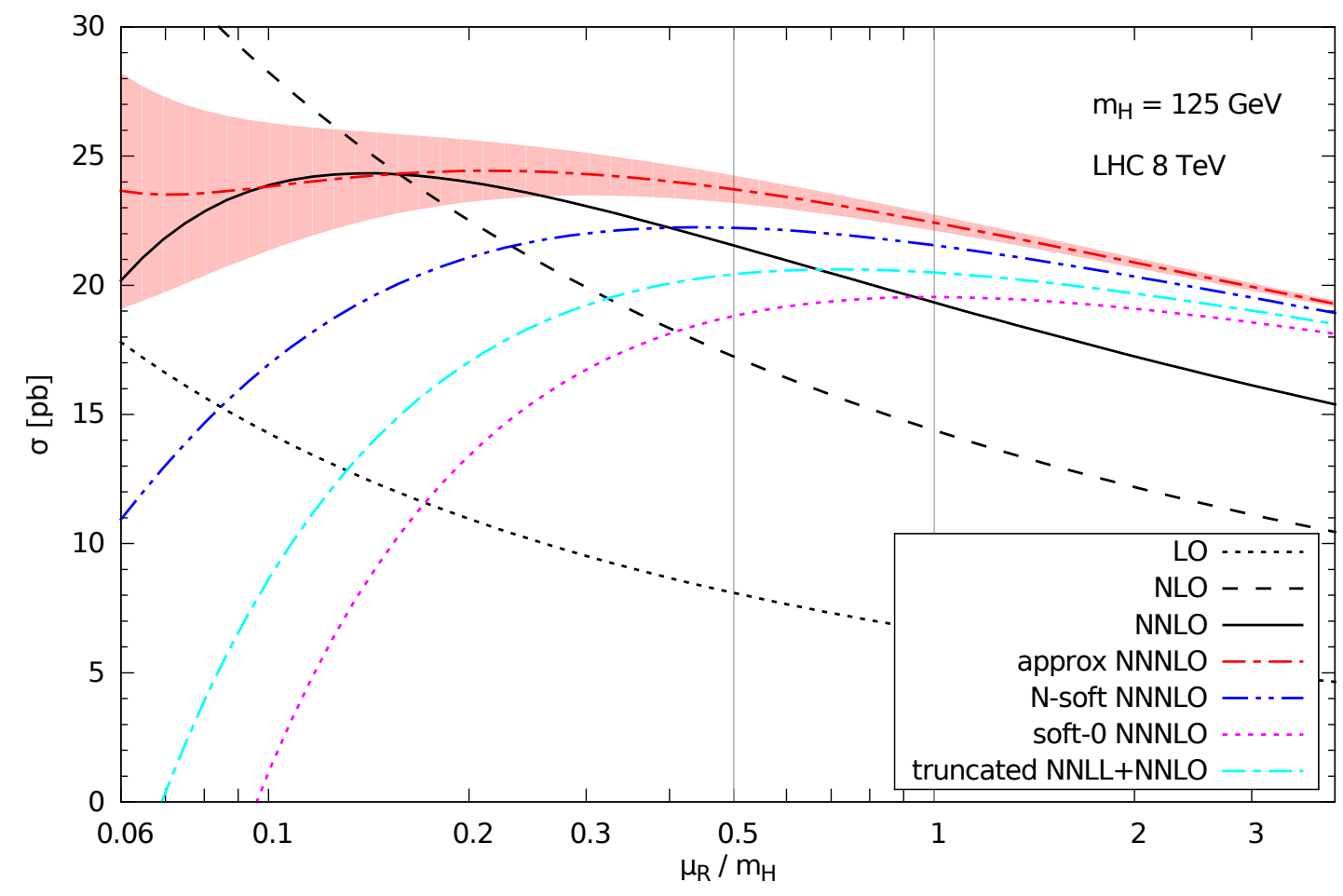

Figure 1. Dependence of the $\mathrm{N}^{3} \mathrm{LO}$ cross section on the renormalization scale $\mu_{\mathrm{R}}$. Two common choices of renormalization scale are shown as vertical bars. The approximate $\mathrm{N}^{3} \mathrm{LO}$ curves are, from top to bottom, our best approximation, the $N$-soft approximation, the $\mathrm{N}^{3} \mathrm{LO}$ truncation of the NNLL resummed result of Ref. 8, and the soft-0 approximation (see text for details). In all cases, the full result with finite top mass is included through NNLO. The known LO, NLO and NNLO results are also shown. The red band provides an estimate of the uncertainty on our result, obtained with the procedure of Ref. [4].

increase of the NNLO by about $6 \%$ at the same scale. Note that the (in principle infinite) series of higher orders included in the resummation only adds an extra $2 \%$ to this.

This truncated NNLL resummed result differs from our approximation in three respects: the value of the constant (which in Ref. [8] corresponds to $g_{0,3}=0$ ); the coefficient of the single-logarithmic term (both the constant and the single log would only appear in next=to-next-to-next-to-leading log $\left(\mathrm{N}^{3} \mathrm{LL}\right)$ resummation); and the fact that the constraints due to matching to high-energy resummation and analyticity are not taken into account. The effect of the single logarithmic term is completely negligible, so the difference is due in roughly equal proportion to each of the other two reasons. This is also illustrated in Fig. 1, the $N$-soft (see Ref. [4] for the precise definition) curve corresponds to using the exact constant (and single-logarithmic term), but otherwise only including in the same form the $\mathrm{N}^{3} \mathrm{LO}$ terms as in the resummation (i.e. without matching and analyticity). This prediction is seen to indeed lie half-way between our approximation and the truncated NNLL resummed result.

Finally, we also show in Fig. 1 the so-called soft-0 approximation (again, see Ref. [4] for a precise definition). This basically amounts to only keeping soft contributions, but in $z$ space rather than in $N$ space, and it would predict a suppression, rather than an enhancement, of the $\mathrm{N}^{3} \mathrm{LO}$ cross section in comparison to the NNLO one, for a wide range of values of $\mu_{R}$. In the soft limit this approximation coincides with the other approximations discussed here, but away from the limit it differs from them by large corrections suppressed by powers of $\frac{1}{N}$ [or $(1-z)$ ]; it is known 4,9 to fail at NLO and NNLO, essentially because it does not respect longitudinal momentum conservation (albeit by subleading terms) [10]. The result found using this soft-0 approximation was explicitly given in Ref. [3]. 
The updated prediction is available through the code ggHiggs (version 1.9 onwards), publicly available at the website http://www.ge.infn.it/ bonvini/higgs/.

\section{Acknowledgments}

We acknowledge useful discussions with Claude Duhr and Franz Herzog on the results of Ref. [3]. The work of SM is supported by the UK's STFC. SF and GR are supported in part by an Italian PRIN2010 grant, and SF also by a European Investment Bank EIBURS grant, and by the European Commission through the HiggsTools Initial Training Network PITN-GA-2012-316704.

\section{References}

[1] A. Djouadi, M. Spira and P. M. Zerwas, Phys. Lett. B 264 (1991) 440.

S. Dawson, Nucl. Phys. B 359 (1991) 283.

M. Spira, A. Djouadi, D. Graudenz and P. M. Zerwas, Nucl. Phys. B 453 (1995) 17 [hep$\mathrm{ph} / 9504378]$.

R. V. Harlander and W. B. Kilgore, Phys. Rev. Lett. 88 (2002) 201801 [hep-ph/0201206].

C. Anastasiou and K. Melnikov, Nucl. Phys. B 646 (2002) 220 [hep-ph/0207004].

V. Ravindran, J. Smith and W. L. van Neerven, Nucl. Phys. B 665 (2003) 325 [hep-ph/0302135].

S. Marzani, R. D. Ball, V. Del Duca, S. Forte and A. Vicini, Nucl. Phys. B 800 (2008) 127 [arXiv:0801.2544 [hep-ph]].

R. V. Harlander and K. J. Ozeren, Phys. Lett. B 679 (2009) 467 [arXiv:0907.2997 [hep-ph]].

R. V. Harlander and K. J. Ozeren, JHEP 0911 (2009) 088 [arXiv:0909.3420 [hep-ph]].

R. V. Harlander, H. Mantler, S. Marzani and K. J. Ozeren, Eur. Phys. J. C 66 (2010) 359 [arXiv:0912.2104 [hep-ph]].

A. Pak, M. Rogal and M. Steinhauser, Phys. Lett. B 679 (2009) 473 [arXiv:0907.2998 [hep-ph]].

A. Pak, M. Rogal and M. Steinhauser, JHEP 1002 (2010) 025 [arXiv:0911.4662 [hep-ph]].

[2] S. Moch and A. Vogt, Phys. Lett. B 631, 48 (2005) [hep-ph/0508265].

P.A. Baikov, K.G. Chetyrkin, A.V. Smirnov, V.A. Smirnov, M. Steinhauser, Phys. Rev. Lett. 102, 212002 (2009) [arXiv:0902.3519 [hep-ph]].

T. Gehrmann, E. W. N. Glover, T. Huber, N. Ikizlerli, C. Studerus, JHEP 1006, 094 (2010) [arXiv:1004.3653 [hep-ph]].

C. Anastasiou, C. Duhr, F. Dulat, B. Mistlberger, JHEP 1307, 003 (2013) [arXiv:1302.4379 [hep-ph]].

T. Gehrmann, M. Jaquier, E. W. N. Glover and A. Koukoutsakis, JHEP 1202, 056 (2012) [arXiv:1112.3554 [hep-ph]].

C. Duhr and T. Gehrmann, Phys. Lett. B 727, 452 (2013) [arXiv:1309.4393 [hep-ph]].

Y. Li and H. X. Zhu, JHEP 1311, 080 (2013) [arXiv:1309.4391 [hep-ph]].

C. Anastasiou, C. Duhr, F. Dulat, F. Herzog and B. Mistlberger, JHEP 1312, 088 (2013) [arXiv:1311.1425 [hep-ph]].

W. B. Kilgore, Phys. Rev. D 89 (2014) 073008 [arXiv:1312.1296 [hep-ph]].

C. Anastasiou, S. Bühler, C. Duhr and F. Herzog, JHEP 1211, 062 (2012) [arXiv:1208.3130 [hep-ph]]. 
M. Höschele, J. Hoff, A. Pak, M. Steinhauser, T. Ueda, Phys. Lett. B 721, 244 (2013) [arXiv:1211.6559 [hep-ph]].

S. Bühler and A. Lazopoulos, JHEP 1310, 096 (2013) [arXiv:1306.2223 [hep-ph]].

K. G. Chetyrkin, B. A. Kniehl and M. Steinhauser, Nucl. Phys. B 510, 61 (1998) [hep$\mathrm{ph} / 9708255]$.

Y. Schroder and M. Steinhauser, JHEP 0601, 051 (2006) [hep-ph/0512058].

K. G. Chetyrkin, J. H. Kuhn and C. Sturm, Nucl. Phys. B 744, 121 (2006) [hep-ph/0512060].

O. V. Tarasov, A. A. Vladimirov and A. Y. .Zharkov, Phys. Lett. B 93, 429 (1980).

S. A. Larin and J. A. M. Vermaseren, Phys. Lett. B 303, 334 (1993) [hep-ph/9302208].

T. van Ritbergen, J. A. M. Vermaseren and S. A. Larin, Phys. Lett. B 400, 379 (1997) [hep$\mathrm{ph} / 9701390]$.

M. Czakon, Nucl. Phys. B 710, 485 (2005) [hep-ph/0411261].

[3] C. Anastasiou, C. Duhr, F. Dulat, E. Furlan, T. Gehrmann, F. Herzog and B. Mistlberger, arXiv:1403.4616 [hep-ph].

[4] R. D. Ball, M. Bonvini, S. Forte, S. Marzani and G. Ridolfi, Nucl. Phys. B 874 (2013) 746 [arXiv:1303.3590 [hep-ph]].

[5] G. Parisi, Phys. Lett. B 90 (1980) 295.

T. O. Eynck, E. Laenen and L. Magnea, JHEP 0306 (2003) 057 [hep-ph/0305179].

V. Ahrens, T. Becher, M. Neubert and L. L. Yang, Phys. Rev. D 79 (2009) 033013 [arXiv:0808.3008 [hep-ph]].

I. W. Stewart, F. J. Tackmann, J. R. Walsh and S. Zuberi, Phys. Rev. D 89 (2014) 054001 [arXiv:1307.1808].

[6] R. D. Ball et al., Nucl. Phys. B 849 (2011) 296 [arXiv:1101.1300 [hep-ph]].

R. D. Ball et al. [The NNPDF Collaboration], Nucl. Phys. B 855 (2012) 153 [arXiv:1107.2652 [hep-ph]].

[7] S. Forte, A. Isgrò and G. Vita, Phys. Lett. B 731 (2014) 136 [arXiv:1312.6688 [hep-ph]].

[8] D. de Florian and M. Grazzini, Phys. Lett. B 718 (2012) 117 [arXiv:1206.4133 [hep-ph]].

[9] S. Catani, D. de Florian, M. Grazzini and P. Nason, JHEP 0307 (2003) 028 [hep-ph/0306211].

[10] S. Catani, M. L. Mangano, P. Nason and L. Trentadue, Nucl. Phys. B 478 (1996) 273 [hepph/9604351]. 